\title{
Innovation Qualité - Neuer Qualitätspreis der SAQM
}

\section{Michelle Gerbera , Fabienne Hohl ${ }^{b}$}

a lic. phil. hum. wissenschaftliche Mitarbeiterin FMH, Abteilung Daten, Demographie und Qualität DDQ / Schweizerische Akademie für Qualität in der Medizin SAQM; ' Abteilung Kommunikation der FMH

Innovation Qualité heisst der neue Qualitätspreis der Schweizerischen Akademie für Qualität in der Medizin SAQM. Er macht das Engagement für praxiserprobte Qualitätsprojekte im Schweizer Gesundheitswesen sichtbar. Reichen Sie bis am 8. Dezember $2017 \mathrm{Ihr}$ Qualitätsprojekt in einer der drei Preiskategorien ein: Patientenversorgung neu gedacht, Patientensicherheit oder Ärzteorganisationen.

Im Schweizer Gesundheitswesen setzen sich zahlreiche Fachpersonen bei ihrer täglichen Arbeit für eine optimale Versorgung von Patientinnen und Patienten ein. Einige davon gehen noch einen Schritt weiter und verwirklichen erfolgreich ihre innovativen Qualitätsideen in kleinen oder grösseren Projekten. Der neue Qualitätspreis Innovation Qualité der SAQM/FMH würdigt dieses grosse Engagement und macht die Erfolge von Vordenkerinnen und Vordenkern der medizinischen Qualität aus verschiedensten Disziplinen und Berufen bekannt.

Während sechs Jahren hat sich die FMH im Rahmen des Swiss Quality Award mit weiteren Trägerorganisationen für die Qualität in der Medizin stark gemacht. Mit der Innovation Qualité lanciert die FMH nun erstmals den Qualitätspreis der vor fünf Jahren gegrün- deten Schweizerischen Akademie für Qualität in der Medizin SAQM. Die Innovation Qualité zeichnet erfolgreiche Initiativen im Bereich Qualität aus und unterstützt diese mit einem Preisgeld von insgesamt 40000 Franken. Der Preis ist breit abgestützt: Er wird von 22 Organisationen (siehe Kasten) des Gesundheitswesens als ideelle Partner mitgetragen.

Verliehen wird die Innovation Qualité alle zwei Jahre, erstmals am 17. April 2018 im Rahmen des SAQMSymposiums in Bern. Die Gewinnerteams stellen dort ihre Projekte einem interessierten Fachpublikum vor und dürfen die Bezeichnung «Gewinner der Innovation Qualité 2018» führen. Die prämierten Qualitätsprojekte werden in der Schweizerischen Ärztezeitung präsentiert sowie den Medien bekannt gemacht.

Ideelle Partner der Innovation Qualité 2018

Die Innovation Qualité 2018 hat folgende ideelle Partner: Bundesamt für Gesundheit BAG - curafutura EQUAM Stiftung - ErgotherapeutInnen-Verband Schweiz EVS - Faculté de biologie et de médecine de I'Université de Lausanne - Faculté de médecine de I'Université de Genève - Föderation Schweizer Psychologinnen und Psychologen FSP - H+ Die Spitäler der Schweiz - Insel Gruppe AG - Medizinische Fakultät der Universität Bern - Nationaler Verein für Qualitätsentwicklung in Spitälern und Kliniken ANQ - Patientenschutz SPO - pharmaSuisse Schweizerischer Apothekerverband - physioswiss - Schweizer Berufsverband der Pflegefachfrauen und Pflegefachmänner SBK - Schweizerische Akademie der Medizinischen Wissenschaften SAMW - Schweizerische Konferenz der kantonalen Gesundheitsdirektorinnen und -direktoren GDK - Schweizerischer Hebammenverband SHV - Schweizerischer Verband der Berufsorganisationen im Gesundheitswesen SVBG - Schweizerischer Verband der Ernährungsberater/innen SVDE - Schweizerischer Verband der Medizinischen Praxis-Fachpersonen SVA - Spitex Schweiz 
Korrespondenz: FMH / Abteilung DDQ Elfenstrasse 18

CH-3000 Bern 15 Tel. 0313591111 saqm[at]fmh.ch

\section{Transparente Beurteilung}

Zwei unabhängige, interprofessionelle Expertenjurys bewerten die Projekte fair und transparent in drei Preiskategorien:

- Patientenversorgung neu gedacht - dotiert mit 15000 Franken (Themenschwerpunkt): Gesucht sind Projekte, welche die Qualität in der alltäglichen Patientenversorgung auf innovative Weise weiterentwickeln.

- Patientensicherheit - dotiert mit 15000 Franken: Die SAQM kürt gemeinsam mit Patientensicherheit Schweiz das beste Projekt zur Förderung der Patientensicherheit.

- Ärzteorganisationen - dotiert mit 10000 Franken: In dieser Preiskategorie prämiert die SAQM das beste Qualitätsprojekt der Berufsorganisationen der Ärzteschaft.
Während die Kategorien Patientenversorgung neu gedacht und Patientensicherheit Fachleuten des gesamten Gesundheitswesens offenstehen, soll die Kategorie Ärzteorganisationen ärztliche Berufsvereinigungen dazu motivieren, ihre Qualitätsprojekte einzureichen.

\section{Jetzt Projekt einreichen!}

Nutzen Sie die Chance und zeigen Fachwelt und Öffentlichkeit, wie Ihr innovatives Projekt zur Weiterentwicklung der Qualität im Gesundheitswesen beiträgt! Bewerben Sie sich bis zum 8. Dezember 2017 für den neuen Qualitätspreis Innovation Qualité. Das Anmeldeformular sowie detaillierte Informationen zum Preis finden Sie unter www.innovationqualite.ch.

\section{Jetzt Projekt einreichen - bis 08.12.2017}

Zukunftsweisende Qualitätsarbeit im Gesundheitswesen gehört aufs Podest: Gewinnen Sie die Innovation Qualité, den neuen Preis für Qualität in der Medizin! Melden Sie Ihr Projekt an auf www.innovationqualite.ch. 